\title{
A New Digital Forensics Model of Smart City Automated Vehicles
}

\author{
Xiaohua Feng \\ School of Computer Science and \\ Technology \\ University of Bedfordshire \\ Luton, UK \\ Xiaohua.feng@beds.ac.uk \\ Tel. +441234400400
}

\author{
Edward Swarlat Dawam \\ School of CST \\ University of Bedfordshire \\ Luton, Beds, UK \\ Edward.Dawam@study.beds.ac.uk
}

\author{
Saad Amin \\ Computer Science Department \\ Coventry University \\ csx188@coventry.ac.uk
}

\begin{abstract}
In the modern world, cyber societies are full of complications. The Internet has brought so many convenient services to our society but Internet is also a mine field. Mass surveillance from smart phone to PC, from automated car to smart television, any online device seems could be turn to privacy breach toolkit.
\end{abstract}

In order to follow the GDPR (General Data Protection Regulation), protect privacy data, including PII (Personally Identifiable Information), against Cyberstalking and many other cybercrime challenges, a novel Digital Forensics Model served for Smart City Automated Vehicles has been developed working on investigating AAV (Autonomous Automated Vehicle) cases. The proposed development is reported to Big Data 2017. Here, we report the update for discussions.

Keywords -Big data, the Cloud security, Smart City monitoring, IoT (Internet of things), PII, Cyberstalking, automated car, Digital Forensics model, GDPR, PPI (Protection of Personal Information), $A A V$

\section{INTRODUCTION}

As a new research area, Digital Forensics is a subject in rapid development. Cyber security for ICT (Information Communications Technology) gets more and more attention. Computing system breaches require Digital Forensics technique to seize the digital evidence to discover who compromised the digital device and what has been carried out maliciously and possible risk and potentials consequence security assessing and many more afterwards. In particular, in attacks on smart cities, Digital Forensics has been facing even more challenge than previous computing or digital system breach investigations.

Smart Autonomous Automated Vehicles (AAVs) are the result of decades of research (beginning from the 1980s) into the field of vehicle automation; an application of smart transportation which is a major feature of smart cities. Their relationship involves the communication of sensor data from vehicles to vehicle (V2V) and vehicle to infrastructure (V2I) with a unified data hub in smart cities so as to aid the relevant authorities in decision making; these sensor data make up the lowest level of smart city infrastructure and are accessed through the smart AAVs Electronic Control Unit/Module (ECU/ECM). Since the ECU is an event data recorder and can be used to aid car forensics in cyber-attack or related accident cases, the information coming from these sensors has to be accurate.

In this work, an investigation and analysis of threats specifically to smart AAVs within a smart city project were carried out. We used a diagnostic tool to connect a laptop to the on-board diagnostic port of modern vehicles, thereby gaining access to the sensor data on the ECU of two different cars using two different on-board diagnostic software tools. The sensor data was imaged and hashed for data integrity, analysed based on the J1979 standard and encrypted for security purpose (SAE, 2012). Recommendations were made on how to acquire, preserve and analyse the car sensor data in a forensics manner based on version 5 of the ACPO (Association of Chief Police Officers) guidelines, leading to the proposal of a forensic model for the investigation of smart AAVs in a smart city context. For many years we have been working on Big Data security analysis (Hashishi, 2011; Feng and Louise, 2013; Liu, 2014), so we carried on Big Data security on Smart City cases.

The Smart City technique makes use of ICT to collect, detect, analyses and integrate the key information data of core systems in running cities. A control center makes intelligent responses to different requirements that include GDPR, daily livelihood, PII security, environmental protection, public safety, PPI, industrial and commercial activities and city services. The Smart City data are too diverse to be dealt with easily. This paper has summarized our creation on a Digital Forensics model. It makes use of a case study of Smart City project with the Autonomous Automated Vehicle services to 
date (Abeykoon, et al. 2017). Further research is proceeding at Cyber Security Laboratory, School of CST, University of Bedfordshire.

\section{BACKGROUND}

Smart city, focuses on the application of the next-generation of information technology to all walks of life, thereby embedding sensors and equipment to power grids, hospitals, roads and railways, bridges and tunnels, dams and water systems, buildings, oil and gas pipelines and other objects in every corner of the world (Mardacany, 2014). This will enable us to integrate the Internet of things through super computers and cloud computing and will also enable people to manage productivity and life more meticulously and more dynamic manner, leading to global intelligence environment.

Smart autonomous automated vehicles (AAVs) is one fundamental application in the field of intelligent/smart transportation systems (ITS/STS). Smart cities make use of the Internet of things, sensor networks and other technological avenues in changing the traditional transport system and establishing the smart traffic management system. Their relationship involves the communication of sensor data between vehicles to vehicle $(\mathrm{V} 2 \mathrm{~V})$ and vehicle to infrastructure (V2I) with a unified data hub in smart cities so as to aid the relevant authorities in decision making. These sensor data make up the lowest level of Smart City infrastructure and are accessed through the smart AAVs Electronic Control Unit/Module (ECU/ECM). Since the ECU is an event data recorder and can be used to aid car forensics in cyber-attack or related accident cases, the information coming from these sensors has to be accurate as it can potentially lead to a lawsuit and subsequent conviction.

Recently, such lawsuits are increasingly dependent on the sensor and event data recorded on the vehicles' electronic control module or electronic control unit (ECM/ECU). These ECM data include speed records, airbag and break light sensor data and other event data that can aid accident reconstructionist to corroborate and classify physical evidence thereby unraveling the true cause of an event. Since these digital data may potentially end up being used as evidence in court, it should be forensically sound. However, practices mostly employed in extracting this information are unprofessional when compared to other areas of digital forensics and therefore require great care on the part of the investigators so as to properly preserve and present evidence.

\section{A. SMART CITY}

According to IBM; Smart City is the use of ICT technologies in collecting data and integrating the key information of core systems in running those cities, while also making intelligent responses to different needs that includes daily livelihood, environmental protection, public safety, industrial and commercial activities and city services.

In 2006, Samuel Palmisano (2006), the IBM CEO made a speech in New York to the foreign relations council. In his speech, he released the concept of "Smart Planet: the agenda of the next generation leaders". This concept is to be subsequently discussed in separate meetings in the US and China in 2009, thereby gaining positive responses from President B. Obama and Prime Minister Wen Jiabao and eventually throughout the world it has gained more acceptances (Su, 2011). However, "Smart City" - initially referred to as wireless city or wireless digital city, has the same approach with "Smart Planet" but only applies to a specific region. It achieves the goal of informational and integrated management of such cities. It is an effective integration of smart planning ideas, smart management methods, smart construction modes, and smart development approaches.

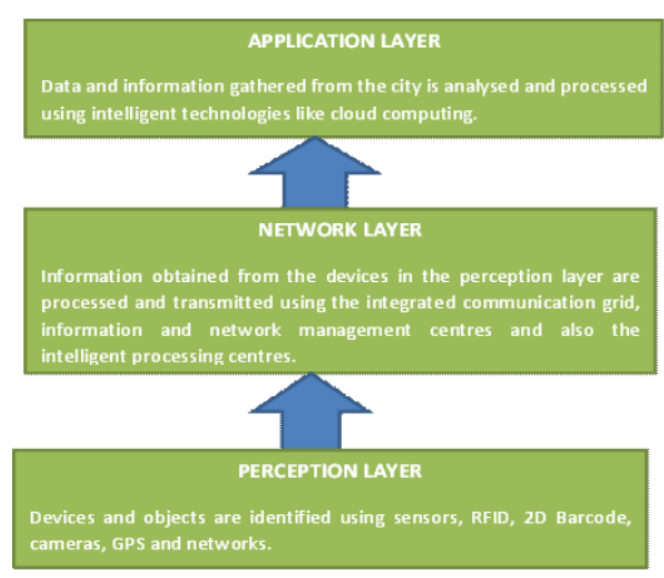

Figure 1 A Smart City Technical Architecture

\section{B. SMART AUTONOMOUS VEHICLES}

According to Hernandes (2013) an Autonomous car/vehicle (in some cases referred to as smart/driverless/self-driving, car /vehicle) "is a self-driving vehicle with the capability of perceiving its environment and can navigate without human intervention. In order to achieve this, complex autonomous driving algorithms that consist of perception, localization, planning and control are required. Several heterogeneous sensors, actuators and computers are also required to achieve autonomous driving".

Kim et al (2014) said that smart autonomous cars have the capability to drive like humans and to make this possible; the car needs to have different components working collaboratively together. These components include; Perception (to evaluate its surroundings), Localization (using the GPS technology), Planning (in order to take the correct action at each step and time.), Control (for the steering, acceleration and breaking) and system management (to supervise the whole vehicle system functionality).

However, the two of them agreed that among the many technologies which are required to achieve autonomous 
automated vehicles are; a combination of actuators and sensors, powerful software executing processors and sophisticated algorithms. It can also be deduced from the both of them, that the sensors and actuators of autonomous cars are categorised into three functions that they aim to achieve: Navigation and guidance sensors - they are responsible for telling you the location, the destination and transportation method. Driving and safety sensors - that are responsible for directing the vehicle to its destination, ensuring that the vehicle acts with precaution in all circumstances and adheres to road and traffic rules. Performance sensors - they manage the basic internal system of the vehicle.

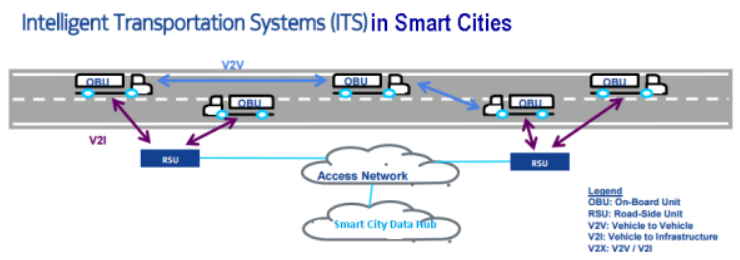

Figure 2 ITS Architecture in a Smart City

Smart autonomous automated vehicles is one of fundamental applications in the field of intelligent/smart transportation systems (ITS/STS). Smart cities make use of the IoT, sensor networks and other technological avenues in changing the traditional transport system and establishing the smart traffic management system. The smart traffic management system is an adaptive traffic signal control system with the capability of automatic control of traffic lights in accordance with the flow and time (Su, 2011). The smart traffic management system also integrates urban planning, construction, management and operations and can further provide a comprehensive support for other subsystems like emission control systems in smart vehicles to monitor and control carbon emission, which is a main aim of Smart Cities.

\section{DIGITAL FORENSICS}

According to Feng et al (2012); "Digital Forensics is a science of acquiring, analysing, extracting, interpreting and producing evidence from a digital source in civil, criminal or cooperate cases of administrative nature". As a result of the growing use of computers and their networks, Digital Forensics has become a vital part of forensics science used in hunting down malicious activities whose traces are mostly found in digital form, thereby aiding in the process of identifying the perpetrators. It evolved from computer forensics, developed further to IoTs, Big Data, the cloud forensics, bio-informatics, PII and many more other area (Hashishi, 2011; Feng, 2012 and 2016).

\section{NEW FORENSICS INVESTIGATION MODEL}

A number of digital forensic investigation models have been proposed, some of them are; Computer forensic investigative process - has four phases; Acquisition, Identification, Evaluation and Admission. (Pollitt, 1995). Digital Forensic Research Workshop (DFRWS) Model has six phases; Identification, Collection, Examination record and Analyses and Presentation (Ismail and Hassan, 2011). Abstract Digital Forensic Model (ADFM), this model was coined out of the DFRWS model and adding three more model phases to it, namely "Preparation, Approach Strategy and Returning Evidence" (Reith and Gunsh, 2002). Integrated Digital Investigation Process (IDIP) consists of five phases, which are; Readiness Phase, Deployment Phase, Physical Crime Scene Phase and Digital Crime Scene Investigation Phase (Ankit, 2011).

From the aforementioned, it can be deduced that all the models share some areas of similarity in the processes of acquisition/collection, preservation, identification, examination/evaluation and presentation. However, the newly Integrated Digital Investigation Process model has shown features that are more relevant to Smart City AAVs as its potential attacks can come from either a physical or cyber source or both and it makes provision for both scenarios.

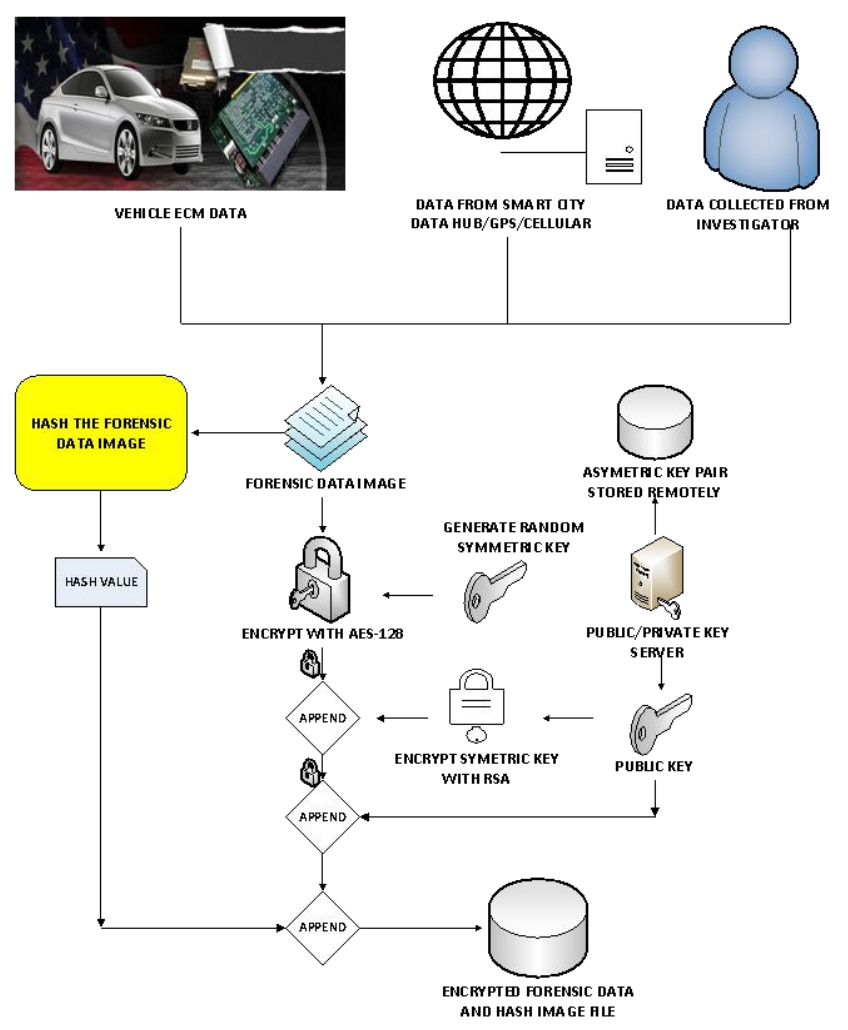

Figure 3 A Novel Forensics Model to Vehicle Data Investigation 


\section{METHODOLOGY}

In this research, we have considered the Smart City AAV to have both cooperative and automated features as it has a very high level of automation in carrying out dynamic driving task. Such that the need of a human driver to monitor threats emanating from the outside environment is not required and it can also perceive its environment using multiple sensors and also make use of the wireless communication technology to perform vehicle-to- $\mathrm{X}$ communication (V2X); where $\mathrm{X}$ is the Smart City road infrastructure which are the stationary road side infrastructures and units which aid smart AAVs. These infrastructure units include traffic signals, map server and road communication unit that broadcasts messages like Road Side Alerts (RSA) and Signal Phase Timing (SPAT), which are some of the typical of Smart City features.

In addition, in the case of AAV investigating, there are emerging errors which is uncommon in conventional Digital Forensics investigations, as shown in Figure 4, therefore made researchers have to carry out the Digital Forensics investigating appropriately for this kind of situations (Woo, 2015; Feng, 2011).

To summarise the experiments in our project, we could reach a conclusion for AVVs as shown in Figure 3. The AAV investigating model in Figure 3 has demonstrated a proposed Digital Forensics investigating approach specially on the Smart City automatic vehicle case process, which including the function of preventing data manipulating by hash verification in order to achieve the data evidence integration as required in principles.

\section{DEVELOPMENT AND EXPERIMENT}

In the Smart City project, Digital Forensics investigation on the AAV case is carried out; for instance, a Laboratory experiment example is demonstrated in Figure 4 and 5.

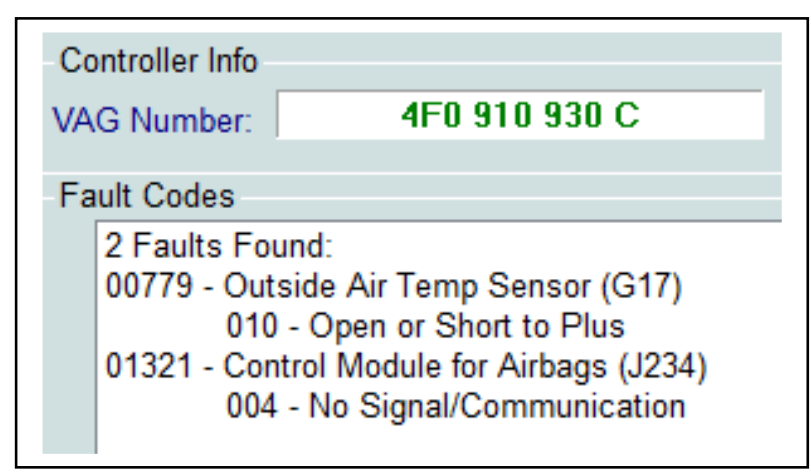

Figure 4 A diagnostic trouble codes of AAV instrument

A special experiment is that an unexpected result shown in Figure 4, which displayed a cluster of diagnostic trouble codes of AAV instrument with errors. To against this kind of error, the model shown in Figure 3 is developed and applied this model to further experiment on investigating Digital Forensics data, the output as shown in Figure 5 below. The output of experiments has demonstrated that the proposed model is successful.

\section{DISCUSSIONS}

This research work has been discussed the vulnerabilities of smart AAVs within the context of a Smart City. It has identified the possible attack vectors and the point of digital evidence collection in smart AAVs. This leads to the identification of the ECM as a prime tool that collects and stores all relevant data associated with a vehicle's operations. This research has progressed further to apply the ACPO guidelines in the handling of incident data contained in a vehicle ECMs. Other concepts like tamper resistance, standard data meanings, transparency and establishing a baseline for participants' trust have been discussed as it relates to the handling of ECM data. An example of Digital Forensics Laboratory experiment is shown in Figure 5.

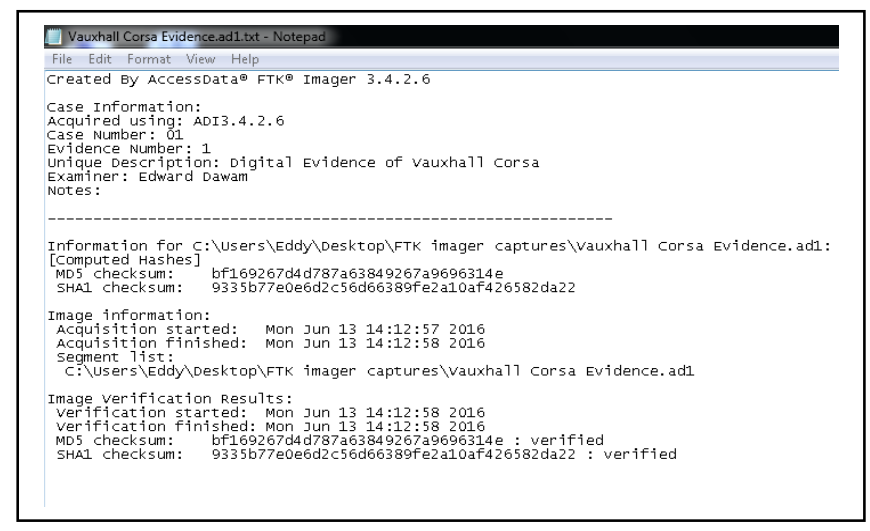

Figure 5 Forensics Lab examples

As the above Figure 5 shown, a demonstration to describe how acquired ECM data/report possibly manipulated is carried out, in order to underpin the research's recommendation that native file formats should not be a trusted format for evidence report.

\section{CONCLUSIONS}

This research has shown that vehicular evidence data can also be extracted, stored and presented in line with the UK ACPO guidelines by using current methods and common tools, but that requires attention and diligence from the investigator. However the issue is that the time vehicles are away from the incident scene as researchers discussed before (Feng, 2016), the data may be altered. Thereby, an inaccurate result might be given by the one which have been acquired from the point 
of an incidence. Furthermore, by identifying all interested parties at the time of evidence extraction is not feasible in some cases. This poses an issue for an investigator using current practices in the absence of witnesses and thus an issue for first responders as well.

Explanations regarding the standards used by diagnostic software and ELM 327 diagnostic adapter are made in a manner that gives meaning to the raw digital data represented in hexadecimal format in the CarScanner and OBD (OnBoard Diagnostics) Auto Doctor diagnostic tools. The level of their compliant with SAE J1979 standard; that defines communication and data interchange between passenger cars, light and medium duty trucks with a wide range of diagnostic test equipment is also discussed and verified. A demonstration of further experimental work and the results regarding the implementation of applying the forensics model to vehicle data details could be provided, represented and discussed.

Future recommendations on the practices to make the forensic processes more reliable and sound were proposed to lead to a development of a digital forensic investigation model on smart AAVs. These recommendations include hashing and encryption of evidence files for integrity purposes, design of diagnostic tools to take activity logs, and so as on. To record an investigators action, to prevent message traffic capable of altering digital record and a replay mechanism replay diagnostic network traffic in order to avoid alteration of the source.

The ease of altering data about evidential files is an issue of concern to vehicle digital data, even though data on the clouds and networks can be trusted (Feng, 2012; Liu, 2014). Also, the access and integrity of data exposed to adverse and damaging conditions is another issue in the design of vehicle ECMs. That is why designers of these systems ought to implement protective measures against intended and accidental alteration of this network data. A sound solution proposed by this research is to have this network data compared with standard external sources, archived, hashed and encrypted to guard against manipulation. This solution should be adopted by all manufacturers that make use of vehicular networks in transmitting data. Immediately after data extraction, the hash value should be distributed to trusted third parties to keep it safely for the further recall.

\section{REFERENCES}

Abeykoon I. Feng X. and Qiu R. (2017) “Acquisition and Recovery Robotics Forensics Evidence", University of Bedfordshire, Research Conference, U.K. 2017.

Altschaffel R, Hoppe T, Kuhlmann S, Dittmann J. (2014) "Beyond mileage". IEEE International Conference on Connected Vehicles and Expo (ICCVE). 22 (4), pp149 - 154.

Anderson Ross (2008) Ross Anderson (2008) "Security Engineering", 2nd Ed. Wiley, ISBN-13: 9780470068526.

Connected Vehicles and Expo (ICCVE). 22 (4), pp. 149 154.
Ankit, A., Gupta, M., Gupta, S. and Prof. Gupta . (2011), "Systematic Digital Forensic Investigation Model". International Journal of Computer Science and Security (IJCSS). 5 (1), pp. 1-14.

Carrier, B. (2005) "Filesystem forensic analysis", Indiana: Addison Wesley Professional, 2005, ISBN: 9780321268174

Cohen, R. (2012) "The past, the present, and the future of cloud computing', Intel Technology Journal, 16 (4), pp. 2024.

Deekue S.; Feng X. and Liu, E (2013) “A strategic framework for Nigeria e-government security", ARSR2013 Workshop, University of Bedfordshire and Manchester, UK

Delport Waldo M. K. and Olivier Martin S. (2011) "Isolating a cloud instance for a digital forensic investigation", proceedings of the Information and Computer Security Architecture (ICSA).

DFRWS (2001) "Workshop 1 - A Framework for Digital Forensic Science". In: G. Palmer, ed. A Road Map for Digital Forensic Research. New York: DFRWS, pp. 15-16.

Dykstra J. and Sherman A. (2011) "Understanding issues in cloud forensics: Two hypothetical case studies," Journal of Network Forensics, vol.b, no. 3, pp. 19-31, 2011.

Feng X. (2016) "Forensics and Cyberstalking “, University of Bedfordshire, UK

Feng X. and Louise, J. (2013) "MITM attack detection on computing networks", The International Journal of Soft Computing and Software Engineering [JSCSE], Vol. 3, No. 3, Special Issue: the Proceeding of International Conference on Soft Computing and Software Engineering 2013 [SCSE'13], San Francisco State University, CA, U.S.A., March 2013 Doi: 10.7321/jscse.v3.n3.78 e-ISSN: 2251-7545

Feng X. (2012) "Cloud computing forensics", Keynote speech, ICFCCT-2012, Beijing China, 2012.

Feng X. (2011) "Incidence Response Strategies", the 7th HEA Annual Forensics Teaching Workshop, Sunderland.

Harshish M and Feng X. (2011) "Challenges on Forensics, A Cloud investigations reference model", STAN-2011, IEEE Symposium of Security, Technology and Networks.

Hernandes A, Brito A. S, Roncancio H, Magalhães D. V, Becker M. Sampaio R. C. B, Jensen B. T. (2013) "GISA: A Brazilian platform for autonomous cars trials". Industrial Technology (ICIT), 2013 IEEE International Conference. 65 (05), pp. $82-87$.

Jo K. Kim J. Kim D. Jang C. and Sunwoo, M. (2014) "Development of Autonomous Car-Part I: Distributed System Architecture and Development Process". IEEE Transactions on Industrial Electronics. 61 (12), pp. 71317140 .

Johnson, J. Daily, J. and Kongs, A. (2014). "On the Digital Forensics of Heavy Truck Electronic Control Modules". SAE International Journal on Commercial Vehicle. 7 (1), pp. 72-88 Liu, E and Feng X. (2014): "Trustworthiness in the Patient Centered Health Care System, Series: Communications in Computer and Information Science, Vol. 426, ISBN 978-3662-43907-4, ISCTCS , ISSN1865-0929, e-ISSN 1865-0937, DOI: 10.1007/978-3-662-43908-1_44, Springer-Verlag Berlin Heidelberg, http://www.springer.com/series, 2014

Palmisano Samule (2006) "Smart planet" IBM talk, http://www.ibm.com/smartplanet/us/en/smart-cities/article (accessed 14/3/2017)

Petit J and Shladover S. (2015). "Potential Cyberattacks on Automated Vehicles". IEEE Transactions on Intelligent Transportation Systems. 16 (2), pp. 546-556.

Pollitt, M. (1995). "Computer Forensics: An approach to evidence in cyberspace". Baltimore: MD. Pp. 487-491.

Reith, C. and Gunsh,C. . (2002) "An Examination of Digital Forenisc Models". International Journal of Digital Evidence, vol. 1 (no. 3 ), pp. 1 - 6 . 
$\begin{array}{lllll}\text { SAE (2012) } & \text { "The } & \text { J1979 } & \text { SAE } & \text { Standard", } \\ \text { http://standards.sae.org/j1979 201202/ (Accessed 14/3/2017) }\end{array}$

$\mathrm{Su} \mathrm{K}, \mathrm{Li} \mathrm{J}$ and $\mathrm{Fu}, \mathrm{H}$. (2011) "Smart city and the applications". Electronics, Communications and Control (ICECC), 2011 International Conference. 10 (2), pp. 1028-31.

Wang Z and Liu Y. (2010) "Design of Road Tracing Navigation Control for Smart Car Use CCD Sensor". IEEE International Conference on E-Health Networking, Digital Ecosystems and Technologies Design. 10 (3), pp. 345-348.

Woo S, Jin Jo H, and Hoon Lee D. (2015) "A Practical Wireless Attack on the Connected Car and Security Protocol for In-Vehicle CAN'. IEEE Transactions on intelligent transportation systems. 16 (2), pp. 993.
Yang, P. Hanneghan, M. Qi, J. Deng, Z. Dong, F. and Fan, D. (2015) "Improving the validity of lifelogging physical activity measures in an IoT environment". IEEE CIT; Ubiquitous Computing and Communications; Dependable, Autonomic and Secure Computing; PICom (CIT/IUCC/DASC/PICOM), International Conference 2015, pp. 2309-2314.

Zawoad Shams and Ragib H. (2013) "Cloud forensics: a meta-study of challenges, approaches, and open problems", University of Alabama at Birmingham, USA. 